\title{
Genome draft of the Arabidopsis relative Pachycladon cheesemanii reveals novel strategies to tolerate New Zealand's high ultraviolet $\mathrm{B}$ radiation environment
}

\author{
Yanni Dong ${ }^{1 \dagger}$, Saurabh Gupta ${ }^{2 \dagger}$, Rixta Sievers $^{3}$, Jason J. Wargent ${ }^{3}$, David Wheeler ${ }^{1}$, Joanna Putterill',
} Richard Macknight ${ }^{5}$, Tsanko Gechev ${ }^{6,7}$, Bernd Mueller-Roeber ${ }^{2,7,8}$ and Paul P. Dijkwel ${ }^{1 *}$ (iD

\begin{abstract}
Background: Pachycladon cheesemanii is a close relative of Arabidopsis thaliana and is an allotetraploid perennial herb which is widespread in the South Island of New Zealand. It grows at altitudes of up to $1000 \mathrm{~m}$ where it is subject to relatively high levels of ultraviolet (UV)-B radiation. To gain first insights into how Pachycladon copes with UV-B stress, we sequenced its genome and compared the UV-B tolerance of two Pachycladon accessions with those of two A. thaliana accessions from different altitudes.

Results: A high-quality draft genome of $P$. cheesemanii was assembled with a high percentage of conserved single-copy plant orthologs. Synteny analysis with genomes from other species of the Brassicaceae family found a close phylogenetic relationship of $P$. cheesemanii with Boechera stricta from Brassicaceae lineage I. While UV-B radiation caused a greater growth reduction in the $A$. thaliana accessions than in the $P$. cheesemanii accessions, growth was not reduced in one $P$. cheesemanii accession. The homologues of $A$. thaliana UV-B radiation response genes were duplicated in $P$. cheesemanii, and an expression analysis of those genes indicated that the tolerance mechanism in $P$. cheesemanii appears to differ from that in $A$. thaliana.
\end{abstract}

Conclusion: Although the $P$. cheesemanii genome shows close similarity with that of $A$. thaliana, it appears to have evolved novel strategies allowing the plant to tolerate relatively high UV-B radiation.

Keywords: Abiotic stress, Arabidopsis, Genome assembly, Pachycladon, UV-B tolerance

\section{Background}

Pachycladon is an allopolyploid genus of the Brassicaceae family with eight perennial species endemic to the South Island of New Zealand and one species to Tasmania (Australia). These Pachycladon species are believed to have originated around 1-3.5 million years ago in New Zealand and are primarily distributed across the alpine regions of the South Island [1, 2]. Pachycladon cheesemanii is the most widespread of the Pachycladon species with a

\footnotetext{
* Correspondence: p.dijkwel@massey.ac.nz

†Yanni Dong and Saurabh Gupta contributed equally to this research and are considered joint first authors.

'School of Fundamental Sciences, Massey University, Tennent Drive,

Palmerston North 4410, New Zealand

Full list of author information is available at the end of the article
}

broad longitudinal distribution in New Zealand and a wide altitudinal range from $10 \mathrm{~m}$ to $1600 \mathrm{~m}$ above sea level [1].

Pachycladon's allopolyploid genome $(2 n=20)$ consists of two subgenomes which resulted from intra- or interspecific crossing [3]. Karyotype comparisons between extant Pachycladon species and the theoretical Ancestral Crucifer Karyotype showed that the chromosome structure had undergone multiple rearrangements prior to the allopolyploidy event taking place [4], and this has hampered efforts to trace back Pachycladon's progenitors. Phylogenetic analysis of Pachycladon species based on five single-copy nuclear genes indicated that one of the genome copies was derived from the Arabidopsis lineage, while another was similar to both Arabidopsis and Brassica lineages [5]. However, a comparison of

(c) The Author(s). 2019 Open Access This article is distributed under the terms of the Creative Commons Attribution 4.0 International License (http://creativecommons.org/licenses/by/4.0/), which permits unrestricted use, distribution, and reproduction in any medium, provided you give appropriate credit to the original author(s) and the source, provide a link to the Creative Commons license, and indicate if changes were made. The Creative Commons Public Domain Dedication waiver (http://creativecommons.org/publicdomain/zero/1.0/) applies to the data made available in this article, unless otherwise stated. 
547 homeologous gene pairs from $P$. cheesemanii and $P$. fastigiatum with the homologous genes from Arabidopsis lyrata and Arabidopsis thaliana found that no set of genes showed significantly different identity to $A$. lyrata and $A$. thaliana homologues, suggesting the two Pachycladon subgenomes are derived from the same lineage [6]. Data from analysis of the nuclear gene CHALCONE SYNTHASE (CHS) further supported the idea that both Pachycladon genome copies stem from the Arabidopsis lineage [7].

Polyploidization has been suggested to contribute to plants' evolution and environmental adaptation under selection pressure [8-10]. Plants with polyploid genomes can benefit from functional diversification of redundant gene copies, with one gene copy retaining the original function, guaranteeing the plant's regular growth and development, while the other can evolve to confer novel phenotypes, such as protection against challenging environmental conditions [11]. Thus, higher levels of UV radiation in New Zealand compared with locations in the Northern Hemisphere at similar latitudes may have contributed to the evolution of the Pachycladon species [12].

$\mathrm{UV}$ radiation is classified into three types, UV-A, UV-B and UV-C. While UV-C does not penetrate the atmosphere, some UV-B radiation reaches Earth's surface, where it can damage important molecules like DNA. In order to acclimate to UV-B radiation, plants have developed multiple strategies, including reducing leaf area by curling of the leaves, inhibiting leaf and plant growth [13, 14] and increasing light reflection by inducing the production of a cuticular wax layer and the biosynthesis of lightabsorbing secondary metabolites $[15,16]$. Nevertheless, excess UV-B radiation can cause the development of hypersensitive response-like necrotic lesions and plant death [17-19].

UV-B radiation is perceived by the UVB-resistance 8 (UVR8) photoreceptor which was discovered by the UV-B hypersensitivity of the uvr8 mutant [20]. The crystal structure of the UVR8 protein showed that its core domain consists of a covalently bound homodimer [21]. After UV-B radiation, this homodimer dissociates and monomeric UVR8 interacts with CONSTITUTIVE PHOTOMORPHOGENIC 1 (COP1) and transcription factors including ELONGATED HYPOCOTYL 5 (HY5) and HY5-HOMOLOG (HYH) to induce the expression of UV-B-responsive genes [22]. Induced genes included those that encode CHS, FLAVANONE 3-HYDROXY LASE (F3H) and FLAVONOL SYNTHASE 1 (FLS1), which are core enzymes involved in the biosynthesis of flavonoids [23] and are believed to function as a UVabsorbing sun screen [24]. Other induced genes include PHOTOLYASE 1 (PHR1), which encodes protein phosphate starvation response 1 , and EARLY LIGHT-INDUCIBLE
PROTEIN1 (ELIP1). ELIP1 plays a role in the interaction of UV-B-induced monomeric UVR8 with chromatin [25]. It was found that the UVR8-dependent pathway responds to a wide range of UV-B radiation $\left(0.1-12 \mu \mathrm{mol} \mathrm{m}^{-2} \mathrm{~s}^{-1}\right)$. Another less well-understood UV-response pathway was found that functions independently of UVR8. By treating uvr8 mutants with relatively high UV-B radiation levels (1$\left.12 \mu \mathrm{mol} \mathrm{m}^{-2} \mathrm{~s}^{-1}\right)$, several genes induced by this pathway were identified [26].

Since $P$. cheesemanii survives in New Zealand's high UV-B radiation environments, this species may have evolved distinct UV-B-radiation response pathways. To learn how this species is able to cope in its unique environment, we first assembled a high-quality draft genome of $P$. cheesemanii and attempted to reveal the two highly similar subgenomes. The draft genome was used to identify $P$. cheesemanii candidate genes likely involved in UV-B radiation response pathways. However, interestingly, the UV-B-induced expression pattern of these genes differed from that observed in two A. thaliana accession with differing UV-B responses, suggesting that a distinct UV-B radiation response pathway has evolved in $P$. cheesemanii to enable adaptation to the high UV-B radiation environment in New Zealand.

\section{Results}

\section{Genome assembly and assessment}

We extracted $P$. cheesemanii Kingston genomic DNA for whole genome sequencing. The Illumina sequencing technology was used to obtain high coverage sequence reads to help us determine its ancestry and current gene-set. Paired-end and mate-pair libraries were sequenced and $\sim 56 \mathrm{~Gb}$ of DNA sequence obtained. Raw reads $(483,792,966$ reads) were subsequently trimmed using the cutadapt algorithm that is present in the trim_galore package. Using $\mathrm{k}$-mer analysis (Additional file 1) the genome size was estimated to encompass $596 \mathrm{Mb}$. Multiple aligners (Platanus and SOAPdenovo) with different k-mer lengths were used to generate genome assemblies. Subsequently, these assemblies were further evaluated using multiple metrics, and the best one (51-k-mer assembly) was selected based on the assembly size and N50 from Platanus (P.k51) (Additional file 2). The assemblies using SOAP resulted in a higher scaffold size compared to Platanus, but also a much higher number of gaps and lower percentage of complete single copy orthologues. Therefore, Platanus was used as the preferred genome assembler. The total assembly size using P.k51 was $422 \mathrm{Mb}$ and this represented $70.8 \%$ of the estimated genome size. The longest scaffold was $418 \mathrm{~kb}$, while the number of scaffolds of length $\geq 500$ and $\geq 1000$ bases were 53,782 and 23,900 , spanning $\sim 300 \mathrm{Mb}$ and 
$\sim 280 \mathrm{Mb}$ of assembly size, respectively. The N50 for the assembly (scaffolds $\geq 500 \mathrm{bp}$ ) was 24,761 bases (Table 1 ). This result indicated that the assembled genome draft was highly fragmented.

A high amount of repetitive DNA in the genome could be one reason for the fragmented genome assembly [27]. Therefore, the repeat content in the genome draft was analyzed using different repeat identification tools, and it was estimated that $\sim 43 \%$ of the total assembly size comprised repeat regions (Additional file 3 and Additional file 4). Among these, $15.96 \%$ were annotated as "retrotransposons", $6.84 \%$ as "DNA transposons" and $19.89 \%$ as "unclassified repeats".

BUSCO assessment revealed that $96.2 \%$ highly conserved plant orthologs were "complete", 1.5\% "fragmented" and 2.3\% "missing". Reads were mapped back to the assembly using Bowtie 2 to show 96.98\% alignment (Table 2). The $P$. cheesemanii leaf transcriptome [6] was aligned against the assembled genome using PASA, and 97.94\% of transcripts could be mapped to the genome (Table 2). A total of 47,821 protein coding genes were predicted using MAKER, with an average transcript size of $1544 \mathrm{bp}$ and 4.42 exons per gene. With regard to noncoding RNAs, 115 rRNA, 707 tRNA and 209 miRNA genes were predicted. In addition, in a comparison of the alleles in P. cheesemanii, 434,467 SNPs and 123,778 SSRs were identified, highlighting the highly polymorphic information content of its genome (Additional file 5). Thus, the results showed a fragmented genome draft, which may be the result of the high number of repeat elements in non-coding regions or/and having two highly similar genomes to contend with. Nevertheless, the assembly of coding regions was deemed of high quality, based on BUSCO and PASA analyses.

\section{Genome functional annotation}

Each of the predicted genes was functionally annotated by using BLASTX against National Center for Biotechnology Information (NCBI) non-redundant protein [28] and Uniprot databases for green plants (Viridiplantae) (Table 3). About $84 \%$ of the predicted genes had a blast

Table 1 Assembly statistics of the $P$. cheesemanii genome

\begin{tabular}{ll}
\hline & Platanus assembly \\
\hline Total assembly size (bp) & $422,560,840$ \\
Number of scaffolds ( $\geq 500 \mathrm{bp})$ & 53,782 \\
Longest scaffold (bp) & 418,003 \\
N50 ( $\geq 500 \mathrm{bp})$ & 24,761 \\
GC (\%) & 36.33 \\
Number of Ns / $100 \mathrm{~kb}(\mathrm{bp})$ & 749.01 \\
Repeats (\%) & 42.96 \\
Number of variants & 434,467 \\
\hline
\end{tabular}

Table 2 Assessment statistics of the $P$. cheesemanii genome

\begin{tabular}{ll}
\hline & Percentage (\%) \\
\hline Read alignment & 96.98 \\
Transcript alignment & 97.94 \\
BUSCO completeness & 96.20 \\
\hline
\end{tabular}

hit against either NCBI nr or Uniprot databases, or against both. Among these, $63 \%$ had a hit in the manually curated Swissprot database. Based on the BLASTX result against NCBI nr, the highest number of hits was with Camelina sativa (24.4\%), followed by Arabidopsis lyrata (22.7\%), Arabidopsis thaliana (19.0\%) and Capsella rubella (17.3\%), all belonging to the Brassicaceae family [29]. InterProScan identified protein signatures for $89.81 \%$ of the predicted proteins, and 2597 genes were classified as transcription factor (TF) encoding genes. Similar to $A$. thaliana, bHLH (239), MYB (212), ERF (211) and NAC (179) TFs comprised the largest TF families in P. cheesemanii. The predicted genes were used for classification into pathways using the KEGG database. Similar to other plant species, the terms "metabolic pathways" and "biosynthesis of secondary metabolites" were assigned to the largest numbers of the predicted genes in $P$. cheesemanii (2930 and 1594, respectively) (Additional file 6).

\section{Synteny analysis of the $P$. cheesemanii genome draft within Brassicaceae species}

It has been reported that the two Pachycladon subgenomes originate from the hybridization of two species of the Brassicaceae family, one each from the Arabidopsis and Brassica lineages [5]. Here, the P. cheesemanii genome was aligned against all publicly available Brassicaceae genomes using MUMmer to perform synteny analysis. Of 28 available Brassicaceae genomes, seven each were from the Brassiceae and Camelineae tribes, four from the Eutremeae tribe, three from the Arabideae tribe, two from the Cardamineae tribe, and one each from the Thlaspideae, Sisymbrieae, Euclidieae, Boechereae, and Aethionemeae tribes (the tribes of Brassicaceae Lineage I: Camelineae, Cardamineae, and Boechereae; the tribes of Lineage II: Sisymbrieae and Brassiceae; the tribe of Lineage III:

Table 3 Annotation statistics of the $P$. cheesemanii genome

\begin{tabular}{ll}
\hline Number of predicted genes & 47,821 \\
Average transcript length (bp) & 1544.46 \\
Average CDS length (bp) & 941.27 \\
Average number of exons per gene & 4.42 \\
Average exon length (bp) & 212.92 \\
Average intron length (bp) & 176.32 \\
Length of scaffolds ( $\geq 500 \mathrm{bp})$ & $299,926,053$ \\
Length of scaffolds $(\geq 1 \mathrm{~kb})$ & $279,782,042$ \\
\hline
\end{tabular}


Euclidieae; the tribes of Expanded Lineage II (EII): Thlaspideae and Eutremeae; the tribe of the basal lineage: Aethionemeae; the unassigned tribe: Arabideae) [29]. Tarenaya hassleriana from the Cleomaceae family was selected as an outgroup [29]. Species with the highest alignment percentage (Maximal Unique Matches: MUMs) against the $P$. cheesemanii genome belong to Boechereae (29\%), Camelineae $(\sim 20 \%)$ and Eutremeae $(\sim 15 \%)$. All pairwise combinations of the Brassicaceae genomes were used to estimate the cumulative alignment percentage with the $P$. cheesemanii genome to determine possible ancestral genomes of Pachycladon. The combination of Boechera stricta and Eutrema heterophyllum had the highest cumulative alignment with $P$. cheesemanii $(37.35 \%)$ at the genome level (Fig. 1a).

From the species with the highest alignment percentage against the $P$. cheesemanii genome, three species from Brassicaceae Lineage I (C. sativa, A. thaliana and $B$. stricta, two from the Camelineae tribe, and one from the Boechereae tribe) and one from Lineage EII (E. heterophyllum, from Eutremeae tribe) [29] were selected for protein ortholog analysis. To identify orthologs, predicted proteins of all five species were blasted against each other in a pairwise manner for a total of 25 combinations. The BLAST searches were further processed using OrthoFinder to identify orthologs. A total of 182,585 genes (76\%) were assigned to 20,553 orthogroups that included 14,971 orthogroups shared within the five species (Fig. 1b). For $P$. cheesemanii, $66.4 \%$ of the genes $(31,749)$ were assigned to $87 \%(17,881)$ of the total orthogroups. Among these orthogroups, 15 novel orthogroups containing 72 genes were present in P. cheesemanii. Based on the orthogroups, a dendrogram of the five species was constructed (Fig. 1c). In accordance with the synteny analysis, $P$. cheesemanii showed the closest relationship with $B$. stricta, followed by $C$. sativa and $A$. thaliana. Beside the orthogroups that were shared by all species, $P$. cheesemanii shared the highest number of orthogroups with C. sativa (2191), followed by B. stricta (1753), A. thaliana (1721) and E. heterophyllum (923). Thus, the data suggests that $P$. cheesemanii has a closer phylogenetic relationship with species from Lineage I of the Brassicaceae family than to those of Lineage EII.

Next, we used the P. cheesemanii, B. stricta, E. heterophyllum and $A$. thaliana genomes to analyze the GO enrichment patterns to further study the phylogenetic relationships of these species. The predicted gene annotations encompassed all major GO terms, suggesting that a core GO term set is present in the $P$. cheesemanii genome annotation (Fig. 2, Additional file 7). A comparison with the GO enrichment distributions of B. stricta, E. heterophyllum and $A$. thaliana revealed a similar pattern across all three GO categories in P. cheesemanii and B. stricta, while the E. heterophyllum pattern was considerably different from the other three species of Brassicaceae Lineage
I (Fig. 2). Therefore, this result provides further support for the closer evolutionary grouping of $P$. cheesemanii with $B$. stricta of Brassicaceae Lineage I, than to E. heterophyllum of Lineage EII.

\section{Different UV-B responses in Pachycladon cheesemanii and Arabidopsis thaliana}

The New Zealand environment is prone to high UV-B radiation levels naturally [30]. We therefore hypothesized that $P$. cheesemanii has evolved a higher UV-B radiation tolerance than its close relative, $A$. thaliana. Two accessions of $P$. cheesemanii were obtained from locations of relatively close proximity to each other. $P$. cheesemanii Kingston was collected just west of Kingston, New Zealand, at an altitude of $\sim 500 \mathrm{~m}$ and $P$. cheesemanii Wye creek was collected $20 \mathrm{~km}$ north of Kingston at an altitude of $\sim 300 \mathrm{~m}$. The $P$. cheesemanii phenotypes were compared against those of the widely studied $A$. thaliana accession Col-0, which grows at an altitude of up to $100 \mathrm{~m}$ (www.arabidopsis. org), and the UV-B-resistant accession Kondara (distribution altitude: $1000-1100 \mathrm{~m}$ ) [31, 32]. To test for responses to UV-B radiation, 28-day-old $A$. thaliana plants and 38-day-old $P$. cheesemanii plants, of similar plant size, were treated with UV-B radiation for 5 days to allow the manifestation of typical UV-B radiation phenotypic responses. A moderately high UV-B radiation $\left(5.2 \mu \mathrm{mol} \mathrm{m}^{-2} \mathrm{~s}^{-1}\right)$ was used to induce both UVR8-dependent and -independent responses.

Leaves of UV-B radiation-treated A. thaliana Col-0 and Kondara plants were significantly smaller than leaves from untreated controls, and the Col-0 accession displayed more necrotic lesions on its leaves than Kondara (Fig. 3a, b, e, f, i, j and Fig. 4a). P. cheesemanii Wye creek plants showed a smaller but significant decrease in leaf size upon UV-B radiation compared to untreated controls. Interestingly, the leaf size of $P$. cheesemanii Kingston was not affected by UV-B radiation (Fig. 4a). All plants displayed some leaf curling and the leaves attained a glossy appearance, which was most apparent in P. cheesemanii Wye creek (Fig. 3c, d, g, h, k, l).

Next, we determined chlorophyll concentration in fully mature leaves of the different accessions. A significant increase in chlorophyll concentration was found in leaves of UV-B radiation-treated A. thaliana Kondara and $P$. cheesemanii Kingston plants, compared to untreated controls, while chlorophyll concentration did not change in A. thaliana Col-0 and $P$. cheesemanii Wye creek plants (Fig. 4b).

Taken together, our results support the notion that $P$. cheesemanii accessions exhibit a higher UV-B radiation tolerance than the A. thaliana accessions. Moreover, the two $P$. cheesemanii accessions responded to $\mathrm{UV}-\mathrm{B}$ radiation in different ways. 
Distinct expression of UV-B radiation-inducible genes in Pachycladon cheesemanii and Arabidopsis thaliana

To further examine the UV-B radiation responses in $P$. cheesemanii and $A$. thaliana, we identified the $P$. cheesemanii homologues of 11 A. thaliana genes that function in the UVR8-dependent pathway and three homologues that play a role in the UVR8-independent pathway. The protein sequences of these genes were used to search the $P$. cheesemanii genome draft using TBLASTN. As a result, at least two potential copies of each gene were

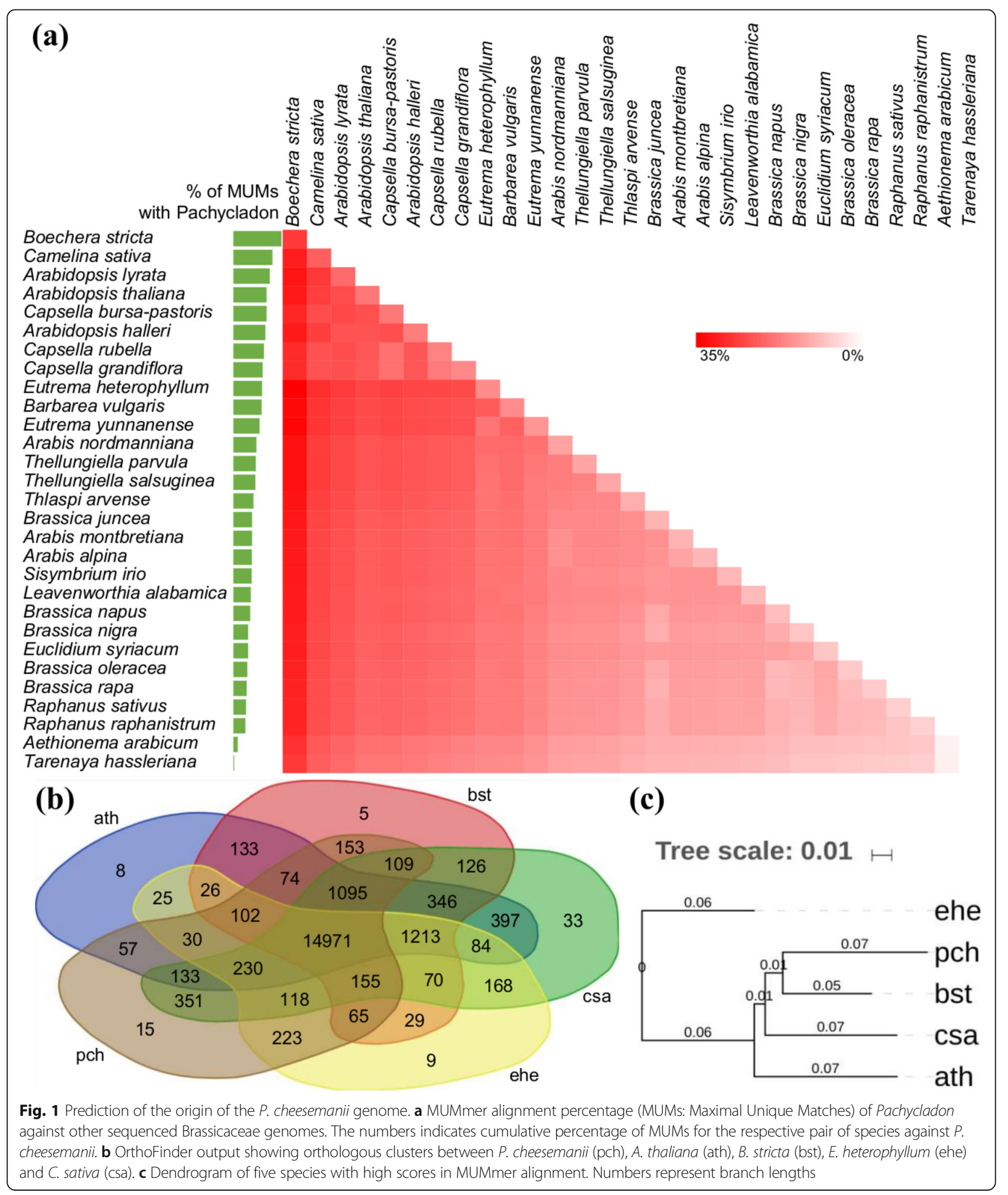




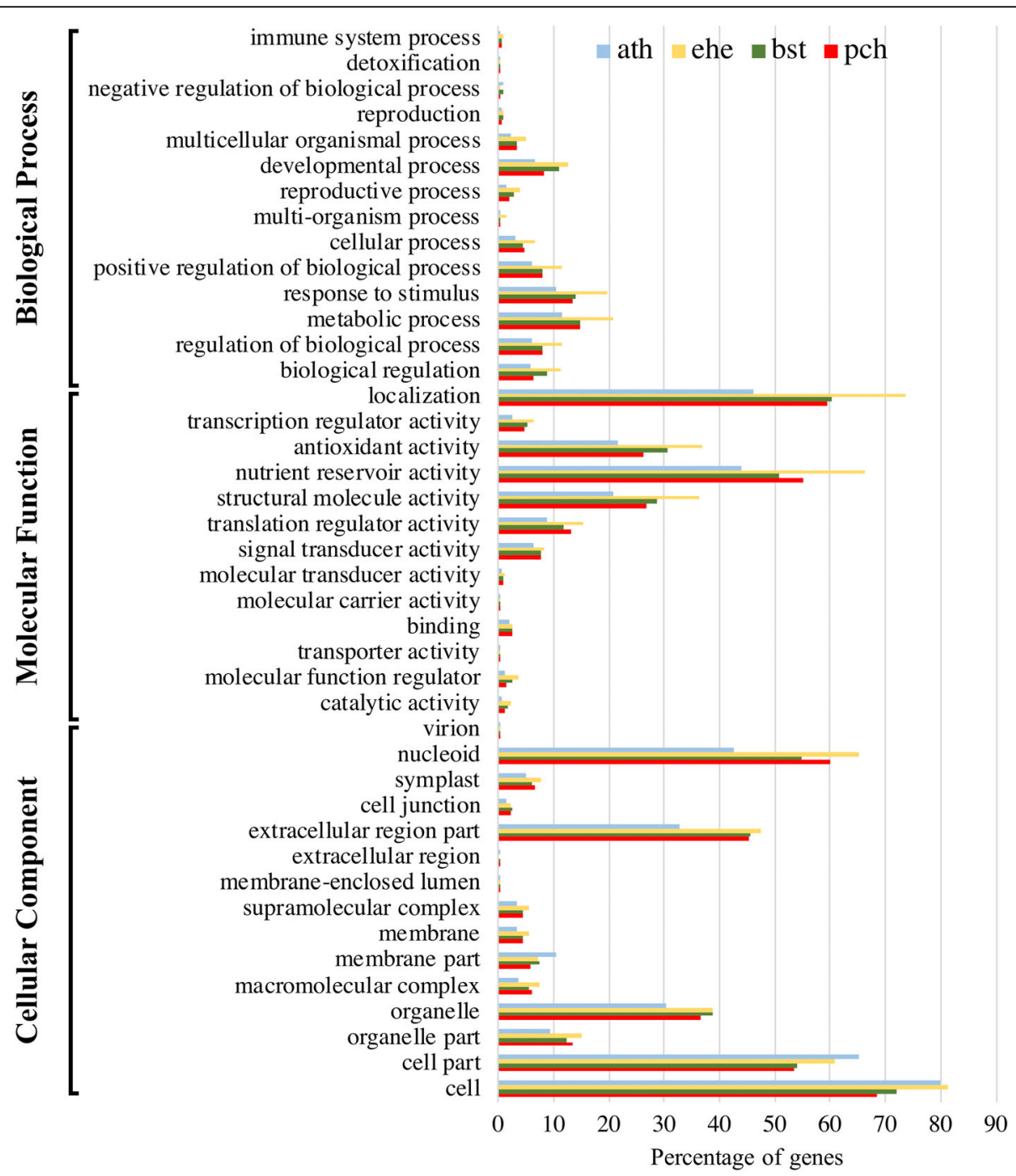

Fig. 2 Gene Ontology (GO) annotation. Comparison of GO terms between P. cheesemanii (pch), A. thaliana (ath), B. stricta (bst) and E. heterophyllum (ehe)

identified (Additional file 8 and Additional file 9), consistent with the polyploid nature of the $P$. cheesemanii genome. Primers for the $P$. cheesemanii genes were designed to amplify conserved protein-coding regions, such that both copies were expected to be amplified with equal efficiency.

$P$. cheesemanii and A. thaliana plants were treated with UV-B radiation for $5 \mathrm{~h}$ to focus on early transcriptional effects and limit secondary responses. Gene expression of the selected genes was measured by quantitative real-time polymerase chain reaction (RTqPCR). We initially measured 11 genes induced in $A$. thaliana by the UVR8-dependent pathway and found that eight (HY5, HYH, CHS, ELIP1, CRYPTOCHROME 3 (CRY3), GLUTATHIONE PEROXIDASE 7 (GPX7), SIGMA FACTOR 5 (SIG5), and WALL-ASSOCIATED RECEPTOR KINASE-LIKE 8 (WAKL8)) were upregulated by UV-B radiation in both $A$. thaliana accessions and three were not $(B C B$, a gene encoding a blue copper binding protein, COP1, and GEM-RELATED 5 (GER5), which encodes a protein involved in hormonemediated regulation of seed germination). Interestingly, while most of these genes were also upregulated in both $P$. cheesemanii accessions, the extent of upregulation was generally lower (Fig. 5).

We next quantified three genes of the UVR8independent pathway, i.e., genes encoding Arabidopsis thaliana WRKY DNA-BINDING PROTEIN 30 (WRKY30), URIDINE DIPHOSPHATE GLYCOSYLTRANSFERASE 74E2 (UGT74E2), and FAD-LINKED OXIDOREDUCTASE (FOX1), and none of those was induced significantly in the A. thaliana accessions by $5.2 \mu \mathrm{mol} \mathrm{m}^{-2} \mathrm{~s}^{-1}$ of UV. However, the WRKY3O homologue was upregulated in both $P$. cheesemanii accessions and the transcript levels of $U G T 74 E 2$ and FOX1 were elevated in $P$. cheesemanii Wye creek, but not in $P$. cheesemanii 


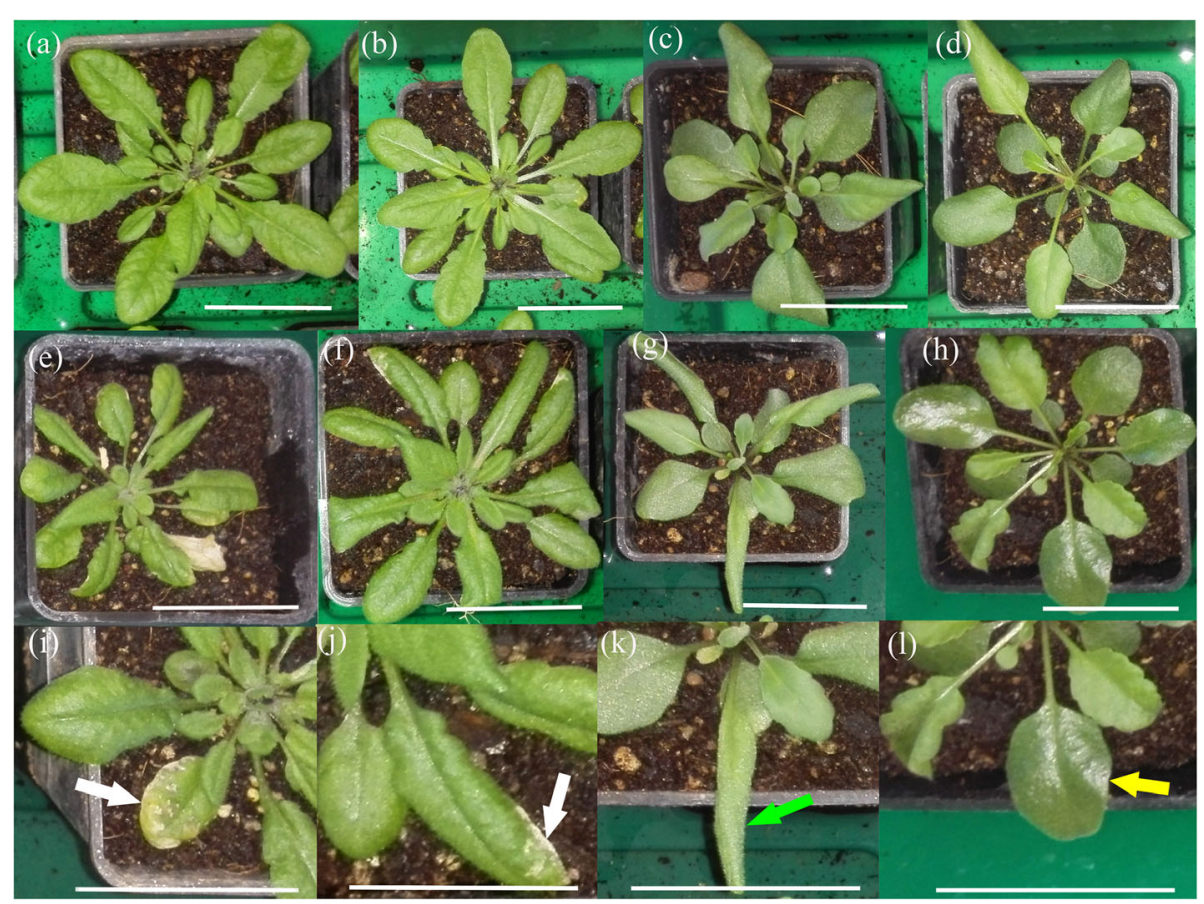

Fig. 3 Twenty-eight-day-old A. thaliana and 38-day-old P. cheesemanii plants after a 5-day UV-B treatment. A. thaliana (28 days old) and P. cheesemanii (38 days old) plants were grown in long day conditions and subsequently transferred to UV-B-supplemented white light for 5 days (UV-B-5-day) or to white light only (control). a A. thaliana Col-0 b A. thaliana Kondara $\mathbf{c} P$. cheesemanii Kingston $\mathbf{d} P$. cheesemanii Wye creek plants grown under control conditions. e $A$. thaliana Col-0 $\mathbf{f} A$. thaliana Kondara $\mathbf{g} P$. cheesemanii Kingston $\mathbf{h} P$. cheesemanii Wye creek plants after UV-B treatment. i-I Enlarged insets are shown for UV-B-treated plants (e-h) only. Arrows indicate necrotic lesions (white), leaf curling (green) and glossy appearance (yellow), respectively. Scale bars, $3.5 \mathrm{~cm}$

Kingston. Thus, A. thaliana and P. cheesemanii accessions responded in different ways to UV-B radiation.

\section{Similar UV-B radiation-repair systems in $P$. cheesemanii} and $A$. thaliana

Plants reduce susceptibility to UV radiation-induced damage through photorepair and dark repair systems
[33]. Here, we identified P. cheesemanii homologues of six key genes involved in UV-B radiation-repair systems in $A$. thaliana. The UV-B radiation-induced transcript level of each gene was subsequently measured in A. thaliana and P. cheesemanii by RT-qPCR. In response to UV-B radiation, the two photorepair genes PHOTOLYASE 1 (PHR1) and UV REPAIR (a)

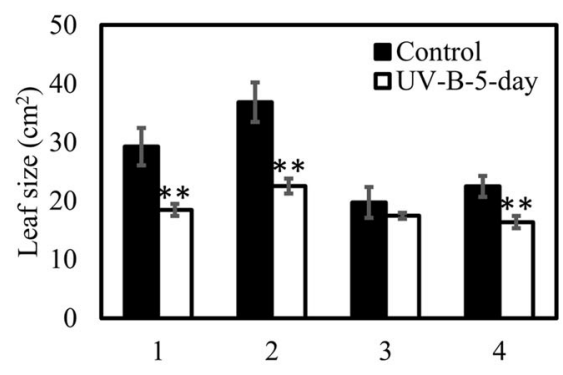

(b)

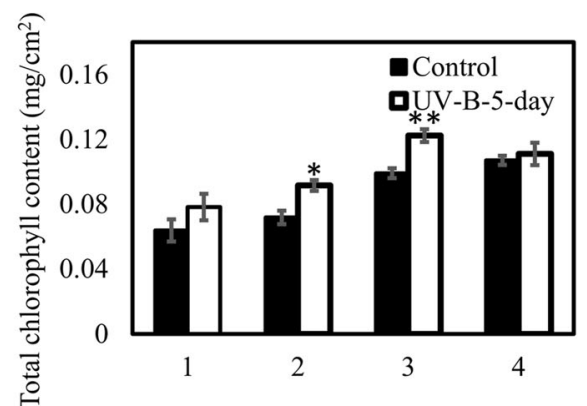

Fig. 4 Total chlorophyll content and leaf size of A. thaliana and P. cheesemanii plants grown with and without UV-B radiation. A. thaliana (28 days old) and $P$. cheesemanii (38 days old) plants were grown in long day conditions and subsequently transferred to UV-B-supplemented white light for 5 days (UV-B-5-day) or to white light only (control). a Total leaf area. b Total leaf chlorophyll content. 1, A. thaliana Col-0; 2, A. thaliana Kondara; 3, P. cheesemanii Kingston; 4, P. cheesemanii Wye creek. Error bars represent SEM (Student's $t$-test; ${ }^{*}, p<0.05 ;{ }^{* *}, p<0.01$ ). Data were collected from 4 to 8 biological replicates 


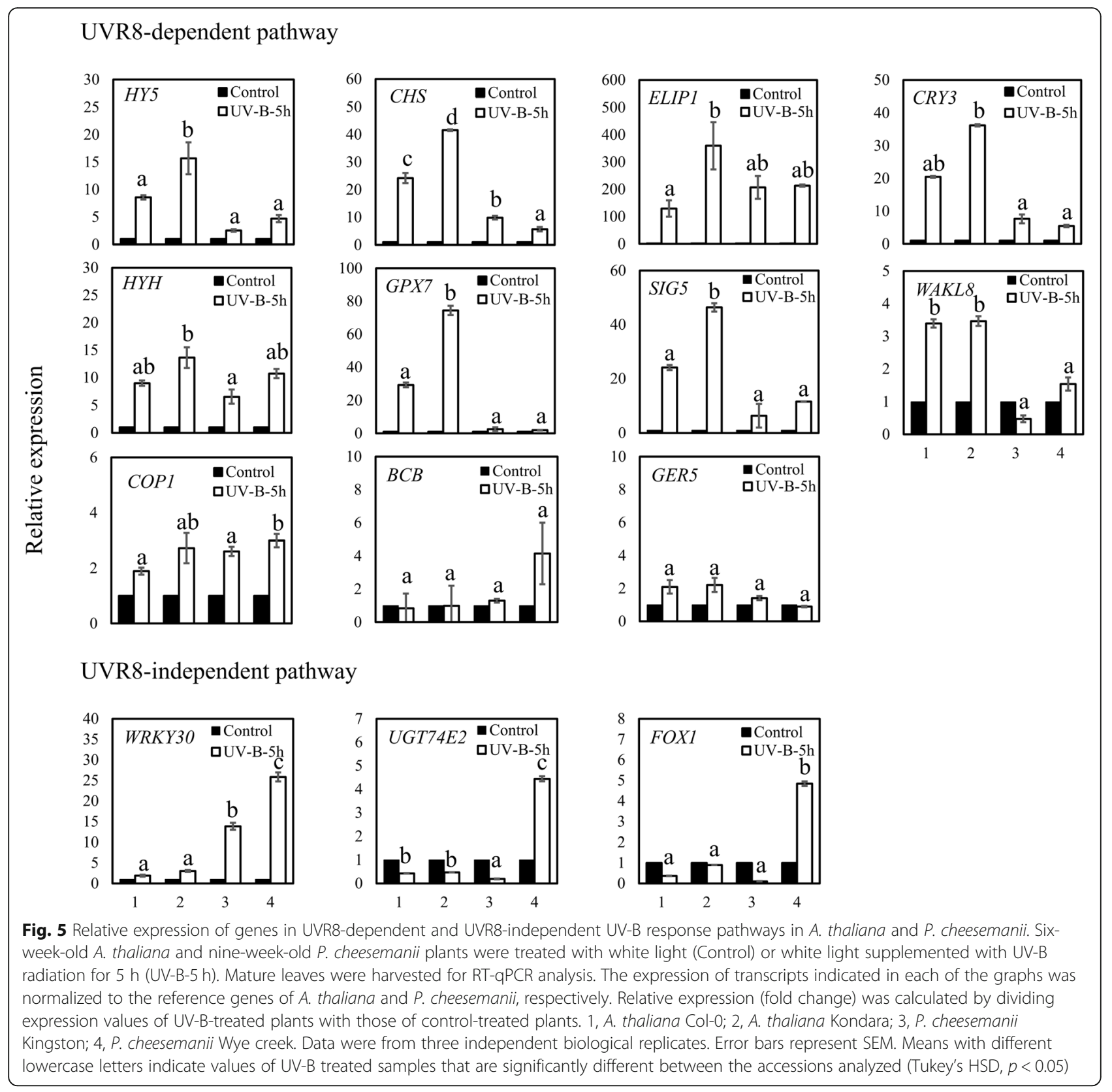

DEFECTIVE 3 (UVR3) were significantly upregulated in all four plant accessions, with A. thaliana Kondara showing the highest increase (Fig. 6). Interestingly, four genes involved in dark repair (nucleotide excision repair) or representing the Arabidopsis homologue of Xeroderma Pigmentosum Complementation Group B1 (XPB1) from humans, i.e., Arabidopsis thaliana XERODERMA PIGMENTOSUM GROUP D (XPD), and a homologue of the human ERCC1 gene (ERCC1), did not show obvious UV-B-induced transcript level changes in $A$. thaliana Col-0 or the two $P$. cheesemanii accessions, while $X P D$ and ERCC1 were upregulated 3.5-fold in A. thaliana Kondara (Fig. 6). Thus, the photorepair genes were upregulated in all plant accessions, while only $A$. thaliana Kondara showed an activated dark repair system.

\section{Discussion \\ Pachycladon cheesemanii may originate from different Brassicaceae lineages}

The two Pachycladon subgenomes have been proposed to result from a relatively young hybridization event, between unknown species, that occurred in New Zealand about 1.61 million years ago [5]. Here, we sequenced the $P$. cheesemanii genome and used it to help trace back the origin of the subgenomes of Pachycladon and to analyze $\mathrm{UV}-\mathrm{B}$ radiation responses. 


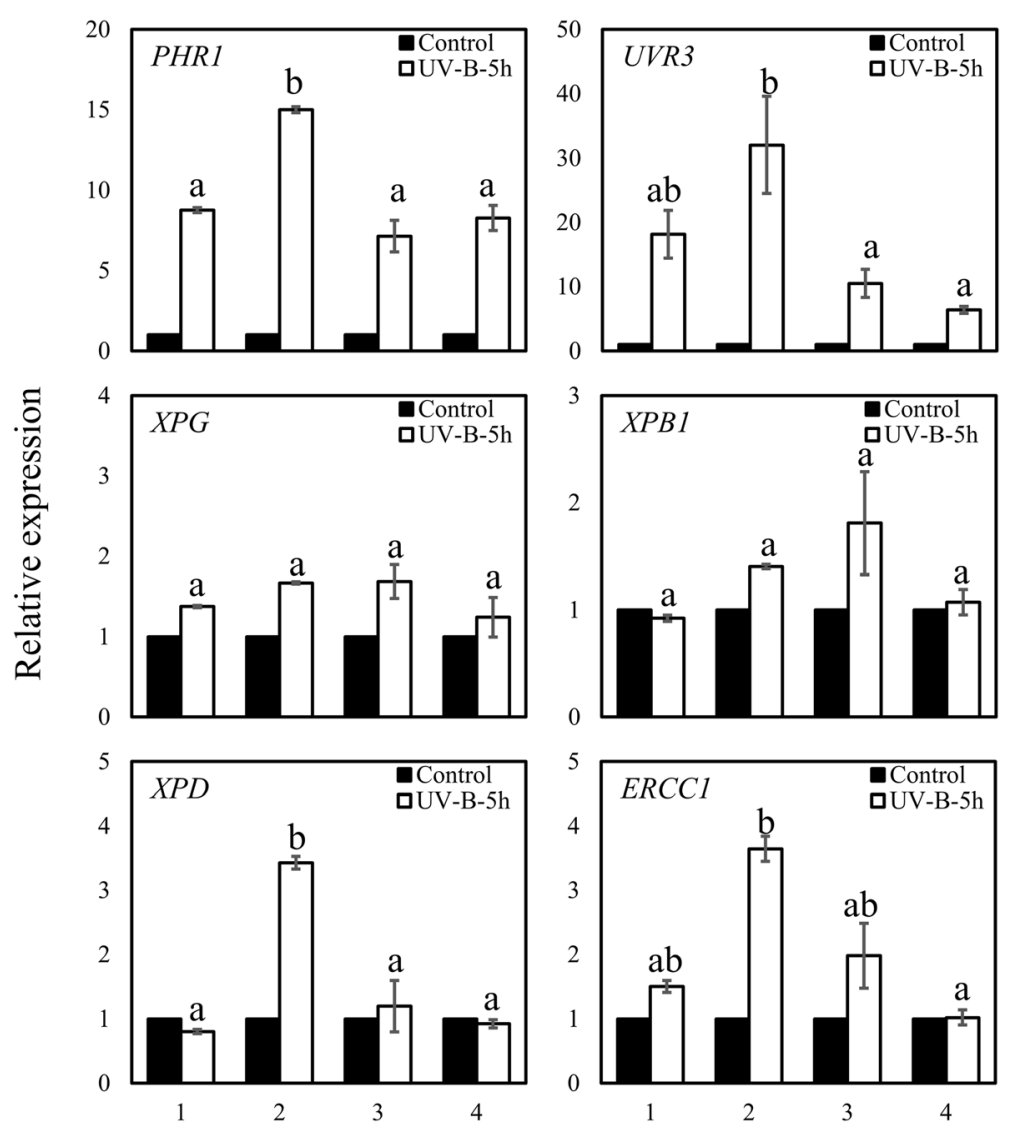

Fig. 6 Relative expression of genes involved in DNA damage repair in A. thaliana and P. cheesemanii. Two genes involved in photorepair (PHR1 and UVR3) and four genes involved in nucleotide excision repair were selected. Six-week-old $A$. thaliana and nine-week-old $P$. cheesemanii plants were treated with white light (Control) or white light supplemented with UV-B radiation for 5 h (UV-B-5 h). Mature leaves were harvested for RTqPCR analysis. The expression of transcripts indicated in each of the graphs was normalized to the reference genes of $A$. thaliana and $P$. cheesemanii, respectively. Relative expression (fold change) was calculated by dividing expression values of UV-B-treated plants with thoes of control-treated plants. Data were from three independent biological replicates. 1, A. thaliana Col-0; 2, A. thaliana Kondara; 3, P. cheesemanii Kingston; 4, P. cheesemanii Wye creek. Error bars represent SEM. Means with different lowercase letters indicate values of UV-B treated samples that are significantly different between the accessions analyzed (Tukey's HSD, $p<0.05$ )

In our synteny analysis of 28 available Brassicaceae genomes, all eight species from tribes Boechereae and Camelineae (genera Arabidopsis, Camelina, Capsella and Boechera) displayed consistent high cumulative genome alignment percentages, regardless as to which of the eight species was combined with any of the 27 other species. Values for Boecherea (B. stricta) were the highest, suggesting that at least one of the $P$. cheesemanii subgenomes originated from the Arabidopsis lineage (Fig. 1a). Nevertheless, no cumulative values higher than $50 \%$ were found, suggesting that only one of the Pachycladon subgenomes has the same origin as these available Brassicaceae genomes. The findings are in general agreement with a recent Brassicaceae phylogenetic tree, established using 113 nuclear genes as markers, which proposes that Pachycladon is closely related to species of Arabidopsis, Camelina, Capsella and Boechera [29]. Similarly, Joly et al. [5] reported that one of the predicted ancestral subgenomes of Pachycladon has close phylogenetic relationships with Boechera and Capsella from the Arabidopsis lineage. However, the origin of the other subgenome is still unclear although phylogenetic analysis of the chloroplast $r b c L$ gene in all Brassicaceae species suggested this genome to be the maternal ancestral subgenome [5]. Heenan et al. [34] proposed that the limited information on Brassicaceae species genomes hampered efforts to determine the origin of the second subgenome, and it may have originated from the tribe Smelowskieae or its close relatives that have subsequently gone extinct [34]. In contrast, we found that the second subgenome might have originated from Brassicaceae Lineage EII. The highest cumulative values were generated when using the combination of B. stricta and E. heterophyllum, the latter of which is from Brassicaceae Lineage EII [29]. The Eutrema tribe was predicted to be 
phylogenetically further away from Pachycladon than the Boechera tribe, however, it is close to the Brassiceae tribe phylogenetically, which could explain the Brassica trace in the Pachycladon genome found previously [5, 29]. Unfortunately, currently little genome information is available for Brassicaceae Lineage EII species, which limits the confident identification of the second Pachycladon subgenome ancestor. Altogether, we concluded that one of Pachycladon subgenomes has an origin similar to that of B. stricta, and that the other possibly arose from Brassicaceae Lineage EII.

\section{Distinct UV-B radiation tolerance responses in $A$. thaliana and $P$. cheesemanii species}

Earlier research has reported that UV-B radiationtolerant plants retained relatively high levels of chlorophyll after UV-B exposure [35]. Consistent with this, a higher chlorophyll concentration was found in UV-B radiation-tolerant $A$. thaliana Kondara as compared to the less UV-B radiation-tolerant accession $A$. thaliana Col-0 (Fig. 4b) [31]. The UV-B response is regulated by at least two pathways of which one is UVR8dependent while the other does not require UVR8 [36]. The UVR8-dependent pathway induces the expression of six genes (CHS, ELIP1, CRY3, GPX7, SIG5, WAKL8) which is mediated by the transcription factors HY5 and $\mathrm{HYH}[26,37]$. Here we found that the genes encoding the transcription factors HY5 and HYH and the six abovementioned genes were induced in response to the UV-B radiation, suggesting that the UVR8-dependent pathway was upregulated in both A. thaliana Kondara and Col-0 accessions. Of note, however, gene expression was generally more induced in A. thaliana Kondara than in Col-0 (Fig. 5). In addition, accession Kondara showed a greater induction of genes involved in photorepair and nucleotide excision repair (Fig. 6), which may allow it to recover from, or prevent UV-B-induced radiation stress [38]. Thus, the induction of chlorophyll biosynthesis genes and a stronger activation of $\mathrm{HY} 5 / \mathrm{HYH}$-pathway genes in Kondara than in Col-0, along with an increased DNA repair capacity in Kondara could be main contributors to its UV$B$ radiation tolerance.

Remarkably, leaf growth of the two P. cheesemanii accessions studied here was less affected by UV-B radiation than that of the A. thaliana accessions Kondara and Col-0; P. cheesemanii Kingston did not show a significant reduction of leaf area upon exposure to UV-B radiation compared to plants grown at control conditions (Fig. 4a). We found that the basal chlorophyll levels in the $P$. cheesemanii accessions were higher than those of the A. thaliana accessions. Furthermore, chlorophyll concentration in $P$. cheesemanii Kingston further elevated upon UV-B radiation and this correlated with its increased tolerance. Chlorophyll levels have been suggested to be an indicator of UV-B radiation tolerance [35] and the observed correlation between UV-B radiation tolerance, chlorophyll levels (Fig. 4b), and the ability to further increase chlorophyll levels upon UV-B radiation is consistent with that suggestion. The $P$. cheesemanii accessions generally induced genes of the UVR8-dependent pathway to a lower extent than the $A$. thaliana accessions, possibly as a result of a higher basal tolerance to UV-B radiation. Brown \& Jenkins [26] identified three genes (WRKY3O, FOX1 and UGT74E2) in A. thaliana that were induced in response to $3-12 \mu \mathrm{mol}$ $\mathrm{m}^{-2} \mathrm{~s}^{-1}$ UV-B radiation as part of the still poorly characterized UVR8-independent pathway. However, in contrast to the $A$. thaliana accessions, both $P$. cheesemanii accessions showed strong induction of the WRKY3O gene at moderately high UV-B radiation levels of $5.2 \mu \mathrm{mol} \mathrm{m}{ }^{-2} \mathrm{~s}^{-1}$. Crucially, only the Wye creek accession of $P$. cheesemanii induced expression of the two other genes (FOX1 and UGT74E2), suggesting that the UVR8-independent pathway may be more important in $P$. cheesemanii than in A. thaliana. Thus, we found a positive correlation between chlorophyll content and UV-B radiation tolerance, and while the stronger induction of the UVR8-dependent pathway in A. thaliana accession Kondara may be at the basis of its UV-B radiation tolerance, the UVR8-independent pathway may play a more prominent role in $P$. cheesemanii accessions. Of further interest is that the lower UV-B radiation tolerance of the Wye creek accession correlated with a much stronger upregulation of genes involved in the UVR8-independent pathway, further supporting that this pathway plays an important role in coping with UV-B radiation damage in $P$. cheesemanii. Indeed, $P$. cheesemanii accessions may be particularly well suited for further exploring the UVR8independent UV-B response pathway helping to increase our understanding of the genomic and genetic basis of plant adaptations to environmental UV-B stress.

\section{Conclusions}

The polyploid nature of the $P$. cheesemanii genome may have contributed to its relatively high tolerance to UV-B radiation. We suggest that tolerance is achieved through the activation of the UVR8-independent response pathway. Our results are important for understanding the allopolyploidy nature of the $P$. cheesemanii genome and may be used to improve UV-B stress tolerance in Brassicaceae crop plants.

\section{Methods}

Plant growth and UV-B treatment

Seeds of $P$. cheesemanii Kingston (geographical coordinates in decimal degrees - 45.3273, 168.7078) and Wye creek (geographical coordinates in decimal degrees $45.1398,168.7672$ ) were provided by Dr. Claudia 
Voelckel (Max Planck Institute for Chemical Ecology, Jena, Germany) and Dr. Peter Heenan (Wildland Consultants Ltd., Rotorua, New Zealand). Seeds of Arabidopsis thaliana (L.) Heynh. accessions Col-0 and Kondara were obtained from the Arabidopsis Biological Resource Center (ABRC; https://abrc.osu.edu). Seeds of both species were sown and germinated in wet Seed Raising Mix soil from Daltons (Matamata, New Zealand) and seedlings were grown under a 16-h light $\left(200 \mu \mathrm{mol} \mathrm{m}^{-2} \mathrm{~s}^{-1}\right.$ cool-white fluorescent tube) $/ 8$-h dark (long-day) regime at $22^{\circ} \mathrm{C}$. For UV-B treatment, the two $P$. cheesemanii accessions were sown 10 days earlier than the $A$. thaliana plants to achieve the same plant size. After 28 (A. thaliana) or 38 (P. cheesemanii) days of growth, the plants were transferred to the UV-B radiation chamber where they were subjected to normal white light $\left(200 \mu \mathrm{mol} \mathrm{m}^{-2} \mathrm{~s}^{-1}\right.$ cool-white fluorescent tube) supplemented with $5.2 \mu \mathrm{mol} \mathrm{m}{ }^{-2} \mathrm{~s}^{-1}$ UV-B (290$320 \mathrm{~nm}$ ) for UV-B treatments, while the control plants were kept under white light conditions. The UV-B fluorescent tubes used in the chamber were Q-Panel 313 (Q-Lab Corp, Cleveland, OH, USA), which were wrapped in 0.13-mmthick cellulose diacetate foil (Clarifoil; Courtaulds Ltd., Derby, UK) to remove wavelengths $<290 \mathrm{~nm}$. The chamber was split into a UV-B+ zone and a UV-B- zone separated by a central curtain of UV-B opaque film (Lumivar; BPI Visqueen, Ardeer, UK). For the UV-B- zone, the UV-B tubes were wrapped in the same UV-B opaque film [39]. UV-B treatments were quantified at plant canopy height with an Optronics OL-756 UV-VIS Spectroradiometer (Optronic Laboratories, Gooch and Housego, FL, USA) equipped with integrating sphere. Spectroradiometric scans of the controlled environment chamber confirmed that the biologically effective UV dose was $<0.01 \mathrm{~kJ} \mathrm{~m}^{-2} \mathrm{~d}^{-1}$ in the UV-B- zone. UV-B-treated and nontreated plants were collected 5 days after the treatment. For quantification of transcript abundance, seedlings were grown under a 10-h light (200 $\mu \mathrm{mol} \mathrm{m}^{-2} \mathrm{~s}^{-1}$ cool-white fluorescent lamp)/14-h dark (short-day) regime at $22^{\circ} \mathrm{C}$ for 6 weeks (A. thaliana) and 9 weeks $(P$. cheesemanii) to obtain a large leaf area to maximise UV-B radiation absorption. The plants were subsequently mock treated or UV-B irradiated for 5 hours, after which leaf tissue was collected.

\section{Library preparation and Illumina sequencing}

Genomic DNA was prepared from P. cheesemanii Kingston leaves. DNA was extracted using a modified CTAB DNA extraction protocol [40] with extra steps to remove chloroplast DNA. Purified P. cheesemanii genomic DNA was used to generate $550-\mathrm{bp}$ paired-end (PE) and 1.5-kb to $15-\mathrm{kb}$ mate-pair (MP) sequencing libraries. Following library quality control, the libraries were sequenced on two lanes of an Illumina HiSeq 2500 machine, with $2 \times 125 \mathrm{PE}$ and $2 \times 125$ MP reads generating a total of $\sim 56 \mathrm{~Gb}$ raw sequencing data. This represents a theoretical coverage of 100-fold based on the estimated size of the $P$. cheesemanii genome (Additional file 10). PE and MP library construction and Illumina sequencing were performed by New Zealand Genomics Limited (NZGL, Otago, New Zealand).

\section{Genome assembly and assessment}

To assess data quality, raw reads were analyzed using SolexaQA++ v3.1.7 [41] (Cox et al., 2010). Adaptors were trimmed using trim_galore v0.4.1 [42], and the processed reads were used for assembly. SOAPdenovo [43] and Platanus v1.2.4 [44] were used for assembly and different k-mer sizes were used to produce multiple assemblies for further quality assessment (Additional file 2). Assembly statistics were generated by QUAST v4.1 and Bowtie 2 $[45,46]$. Leaf transcriptome data (full transcripts) of $P$. cheesemanii [6] (downloaded from NCBI) were used for mapping to the genome assemblies using PASA v2.0.2 [47]. BUSCO v3.0.2 [48] (dataset: "embryophyta_odb9" containing 1440 orthogroups, downloaded from http:// busco.ezlab.org) was used to evaluate the completeness of the assemblies. The result metrics from the tools mentioned above were used to select the best assembly (Additional file 2). Samtools [49] was used for identifying SNPs. After quality filtering, the approximate genome size was determined using the total base number of sequencing data and sequencing depth being derived from the kmer distribution using the formula [50]: $\mathrm{G}=(\mathrm{N} \times(\mathrm{L}-\mathrm{K}+$ 1)-B)/D; G, genome size; $N$, number of reads; $L$, length of reads; $K$, length of $k$-mer; $B$, low-frequency $k$-mers with occurrence less than four times; $\mathrm{D}$, sequencing depth corresponding to selected $\mathrm{k}$-mer.

\section{Identification of repeats}

RepeatModeler v1.0.8 [51], employing RepeatScout, Tandem Repeats Finder and RECON modules, was used to construct a de novo repeats library for $P$. cheesemanii. LTR_finder v1.0.5 [52], TransposonPSI v08222010 [53], and MITE-hunter v11-2011 [54] were used to identify Long Terminal Repeats (LTRs), retrotransposons, and Miniature Inverted repeat Transposable Elements (MITEs), respectively. Viridiplantae repeats were extracted from Repbase repeat libraries [55]. The assembly was masked by de novo and Viridiplantae repeats using RepeatMasker v4.0.5 [56] to estimate the non-redundant genomic repeat content. The repeats were further classified using the RepeatClassifier module of RepeatModeler.

\section{Functional annotation}

A combination of $a b$ initio and homology-based annotation methods was used in the Maker pipeline v2.31.8 [57] for gene prediction. Briefly, AUGUSTUS v3.2.2 [58] was used with an Arabidopsis training set and leaf transcriptome alignments from PASA were used to improve and provide evidence for gene prediction. Gene annotation was done 
using BLASTP v2.6.0 [59] and BLASTX against Uniprot (Swissprot + TrEMBL) and the NCBI nr database (only BLASTX), restricting the search to Viridiplantae using taxonomy filter, best hit, E-value cutoff of $1 \mathrm{e}-20$, query coverage of $\geq 50 \%$ and percentage identity $\geq 50 \%$. Annotations for each gene were selected as per the following order of preferences: 1) Swissprot (BLASTP), 2) Swissprot (BLASTX), 3) Uniprot (BLASTP), 4) Uniprot (BLASTX), 5) NCBI-nr (BLASTX). InterProScan v5.22-61.0 [60] was used for function annotation of the protein domains and families using all available databases in InterProScan. GO annotations were obtained from Uniprot database. Transcription factors were predicted using PlantTFDB v4.0 [61]. KAAS [62] was used to obtain KEGG pathway annotations. MISA [63] was used to predict SSR markers. Infernal v1.1.2 [64] was used to predict non-coding RNAs with an E-value cutoff of 1e-5 and removing low-scoring overlaps.

\section{Comparative genomics}

Genomes of available Brassicaceae species were downloaded from NCBI and Phytozome [65]. Nucmer from MUMmer v3.23 [66] was used to align P. cheesemanii genome sequences against each Brassicaceae genome to perform synteny analysis. Delta filter was applied to select only one-to-one alignments. OrthoFinder v2.1.2 [67] was used to find orthologs among the protein sequences of the selected genomes. GO annotations for A. thaliana were obtained from Araport11 [68], and for B. stricta and $C$. sativa, the GO annotations were obtained in the same way as for $P$. cheesemanii. The dendrogram was plotted using iTOL [69]. Overlaps between orthogroups were plotted using the ClusterVenn utility in OrthoVenn [70].

\section{Chlorophyll content measurement}

Chlorophyll content was measured by using a modified chlorophyll analysis protocol based on Ignat et al. [71]. Two fresh mature leaves were immersed in $10 \mathrm{ml} 95 \%$ ethanol, and tubes were placed in a cool room $\left(4^{\circ} \mathrm{C}\right)$ for $24 \mathrm{~h}$. The chlorophyll-extraction solution was then collected into new tubes, and a further $10 \mathrm{ml}$ ethanol was added to the leaves for another $4 \mathrm{~h}$ to collect the remainder of the chlorophyll. The combined $20 \mathrm{ml}$ chlorophyll-extraction solution was mixed well and $2 \mathrm{ml}$ was used to quantify the chlorophyll content at 664 and $649 \mathrm{~nm}$ by using the following formulas [71]:

$$
\begin{aligned}
& \text { Chlorophyll } a=(13.36 \times A 664-5.19 \times A 649) \\
& \text { Chlorophyll } b=(27.43 \times A 649-8.12 \times A 664)
\end{aligned}
$$

$$
\text { Total chlorophyll }=(5.24 \times A 664+22.24 \times A 649)
$$

Subsequently, chlorophyll contents were normalized to the corresponding total leaf areas that were used to extract chlorophyll.
RNA extraction, CDNA synthesis and RT-qPCR

Total RNA was extracted from mature leaves with a Quick-RNA MiniPrep Kit (Zymo Research, CA, USA) and treated with DNase I to remove genomic DNA contamination. Reverse transcription was performed with an oligo (dT) primer using a Transcriptor First Strand cDNA Synthesis Kit (Roche, Basel, Switzerland). The gene sequences and primers used in RT-qPCR are listed in Additional files 9 and 11. RT-qPCR was performed using a LightCycler 480 SYBR Green I Master kit (Roche) (LightCycler 480; Roche). For real-time PCR quantification, AT4G29130 was used as a reference gene in A. thaliana, and the homologue of AT4G34270 was used as a reference gene in $P$. cheesemanii based on previous reports $[72,73]$. The $P$. cheesemanii homologue of AT4G34270 was identified by applying TBLASTN.

\section{Supplementary information}

Supplementary information accompanies this paper at https://doi.org/10. 1186/s12864-019-6084-4.

Additional file 1. K-mer analysis for estimating the genome size of $P$. cheesemanii. The genome size was estimated by using the formula: $G=(N \times(L-K+1)-B) / D . G$, genome size; $N$, number of reads; $L$, length of reads; $K$, length of $\mathrm{K}$-mer; $\mathrm{B}$, low-frequency $\mathrm{k}$-mers with occurrence less than four times; $D$, coverage depth corresponding to selected k-mer. $41,51,61,71,81,91$, and 101-mer sizes were analyzed, and the coverage depth of 41-mer was selected for genome size estimation.

Additional file 2. Statistics of different assemblies for Pachycladon cheesemanii.

Additional file 3. Repeats content estimation and classification for Pachycladon cheesemanii.

Additional file 4. Characteristics of the 20 longest scaffolds of the $P$. cheesemanii genome assembly. 1) SNPs, 2) GC content (bin: 5 kb; axis: 0$50 \%), 3$ ) repeats, 4) read coverage (bin: $5 \mathrm{~kb})$, 5) predicted genes, 6) PASA alignments against the leaf transcriptome, 7) distribution of gaps (Ns).

Additional file 5. Distribution of Simple Sequence Repeats (SSR) markers identified using MISA.

Additional file 6. KEGG pathway annotation for all genes obtained from KEGG Automated Annotation Server (KAAS).

Additional file 7. Gene Ontology annotation for all genes obtained from BLAST similarity searches.

Additional file 8. Comparison of transcript and genomic DNA sequences between $A$. thaliana CHS and two $P$. cheesemanii homologues. The two $P$. cheesemanii homologues show obvious sequence differences from $A$. thaliana, and slight differences between them. Full sequences of the $P$. cheesemanii genes are given in Table S3. The indicated sequences were aligned using CLUSTAL OMEGA (1.2.4) (https://www.ebi.ac.uk/Tools/msa/ clustalo/) and the conserved nucleotide sequences are indicated by asterisks below the sequences.

Additional file 9. Sequences of genes used for RT-qPCR analysis. Additional file 10. Read statistics of libraries used for sequencing. Additional file 11. List of primers used for RT-qPCR analysis.

\section{Abbreviations}

ABRC: Arabidopsis Biological Resource Center; BCB: Blue Copper Binding protein; CHS: Chalcone Synthase; COP1: Constitutive Photomorphogenic 1; CRY3: Cryptochrome 3; Ell: Expanded Lineage II; ELIP1: Early Light-Inducible Protein 1; ERCC1: Excision Repair 1; F3H: Flavanone 3-Hydroxylase; FLS1: Flavonol Synthase 1; FOX1: FAD-linked Oxidoreductase 1; GER5: GemRelated 5; GPX7: Glutathione Peroxidase 7; HY5: Elongated Hypocotyl 5; 
HYH: HY5-Homolog; KAAS: KEGG Automated Annotation Server; LTRs: Long Terminal Repeats; MITEs: Miniature Inverted repeat Transposable Elements; MP: Mate-Pair; MUMs: Maximal Unique Matches; NCBI: National Center for Biotechnology Information; NZGL: New Zealand Genomics Limited; PE: Paired-End; PHR1: Photolyase 1; RT-qPCR: Quantitative Real-Time Polymerase Chain Reaction; SIG5: Sigma Factor 5; SSR: Simple Sequence Repeat; TF: Transcription Factor; UGT74E2: Uridine Diphosphate Glycosyltransferase 74E2; UV-A: Ultraviolet A; UV-B: Ultraviolet B; UV-C: Ultraviolet C; UVR3: UV Repair defective 3; UVR8: UVB-Resistance 8; WAKL8: Wall-Associated receptor Kinase-Like 8; WRKY30: WRKY DNA-binding protein 30; XPB1: Xeroderma Pigmentosum complementation group B1; XPD: Xeroderma Pigmentosum group D

\section{Acknowledgements}

Authors thank Dr. Christian Kappel (University of Potsdam, Potsdam, Germany) for providing infrastructure for bioinformatics analysis, Dr. Claudia Voelckel (MPI for Chemical Eology, Jena, Germany) and Dr. Peter Heenan (Wildland Consultants Ltd., Rotorua, New Zealand) for providing Pachycladon cheesemanii seeds. The authors would also like to acknowledge the sequencing support from NZGL.

\section{Authors' contributions}

PD, JP and RM conceived the idea. PD, JP, RM, TG and BM-R designed the study. YD and SG performed the computational analysis of the Pachycladon sequence data. YD performed the experiments with Pachycladon and $A$. thaliana accessions. RS and JW designed the UV-B experiments. DW supervised data analysis. PD, RM, BM-R and TG supervised the research. YD, SG, BM-R and PD wrote the manuscript, which was corrected and approved by all authors.

\section{Funding}

This project has received funding from the European Union's Horizon 2020 research and innovation programme under grant agreement no. 642901 (CropStrengthen) and project PlantaSYST (SGA-CSA No. 739582 under FPA No. 664620). The funding bodies did not participate in the study design, data analysis, data collection, interpretation and manuscript writing.

\section{Availability of data and materials}

Sequencing data are available from the NCBI Bioproject database (www.ncbi. nlm.nih.gov/bioproject) under ID: PRJNA475190.

\section{Ethics approval and consent to participate}

Not applicable.

\section{Consent for publication}

Not applicable.

\section{Competing interests}

The authors declare that they have no competing interests.

\section{Author details}

${ }^{1}$ School of Fundamental Sciences, Massey University, Tennent Drive, Palmerston North 4410, New Zealand. ²Department Molecular Biology, Institute of Biochemistry and Biology, University of Potsdam, Karl-Liebknecht-Straße 24-25, Haus 20, 14476 Potsdam, Germany. ${ }^{3}$ School of Agriculture \& Environment, Massey University, Palmerston North 4442, New Zealand. ${ }^{4}$ School of Biological Sciences, University of Auckland, Auckland, New Zealand. ${ }^{5}$ Biochemistry Department, School of Biomedical Sciences, University of Otago, Dunedin, New Zealand. ${ }^{6}$ Department of Plant Physiology and Molecular Biology, University of Plovdiv, 24 Tsar Assen str, 4000 Plovdiv, Bulgaria. ${ }^{7}$ Center of Plant Systems Biology and Biotechnology (CPSBB), 139 Ruski Blvd, 4000 Plovdiv, Bulgaria. ${ }^{8}$ Max Planck Institute of Molecular Plant Physiology, Am Mühlenberg 1, 14476 Potsdam, Germany.

\section{Received: 16 April 2019 Accepted: 6 September 2019}

Published online: 12 November 2019

\section{References}

1. Heenan P, Mitchell A. Phylogeny, biogeography and adaptive radiation of Pachycladon (Brassicaceae) in the mountains of South Island, New Zealand. J Biogeogr. 2003;30:1737-49.
2. Yogeeswaran K, Voelckel C, Joly S, Heenan P. Pachycladon. In: Kole C, editor. Wild crop relatives: genomic and breeding resources wild relatives of oilseeds. Tokyo: Springer; 2011. p. 227-49.

3. Song Q, Chen ZJ. Epigenetic and developmental regulation in plant polyploids. Curr Opin Plant Biol. 2015;24:101-9.

4. Mandakova T, Heenan PB, Lysak MA. Island species radiation and karyotypic stasis in Pachycladon allopolyploids. BMC Evol Biol. 2010;10:367.

5. Joly S, Heenan PB, Lockhart PJ. A Pleistocene inter-tribal allopolyploidization event precedes the species radiation of Pachycladon (Brassicaceae) in New Zealand. Mol Phylogenet Evol. 2009;51:365-72.

6. Gruenheit N, Deusch O, Esser C, Becker M, Voelckel C, Lockhart P. Cutoffs and k-mers: implications from a transcriptome study in allopolyploid plants. BMC Genomics. 2012:13:92.

7. Zhao B, Liu L, Tan D, Wang J. Analysis of phylogenetic relationships of Brassicaceae species based on Chs sequences. Biochem Syst Ecol. 2010:38:731-9.

8. Bowers JE, Chapman BA, Rong J, Paterson AH. Unravelling angiosperm genome evolution by phylogenetic analysis of chromosomal duplication events. Nature. 2003;422:433-8.

9. Brochmann C, Brysting A, Alsos I, Borgen L, Grundt H, Scheen A-C, Elven R. Polyploidy in arctic plants. Biol J Linn Soc. 2004;82:521-36.

10. Wood TE, Takebayashi N, Barker MS, Mayrose I, Greenspoon PB, Rieseberg $\mathrm{LH}$. The frequency of polyploid speciation in vascular plants. P Natl Acad Sci USA. 2009:106:13875-9.

11. Comai L. The advantages and disadvantages of being polyploid. Nat Rev Genet. 2005;6:836-46

12. McKenzie R, Liley B, Kotkamp M, Shiona H, Lopez L. Long term changes in UV in New Zealand due to ozone depletion and other causes. Auckland: NIWA UV Workshop; 2014. p. 15-7.

13. Nascimento LBS, Moreira NS, Leal-Costa MV, Costa SS, Tavares ES. Induction of wound-periderm-like tissue in Kalanchoe pinnata (lam.) Pers. (Crassulaceae) leaves as a defence response to high UV-B radiation levels. Ann Bot-London. 2015:116:763-9.

14. Qaderi MM, Yeung EC, Reid DM. Growth and physiological responses of an invasive alien species, Silene noctiflora, during two developmental stages to four levels of ultraviolet-B radiation. Ecoscience. 2008:15:150-9.

15. Steinmüller D, Tevini M. Action of ultraviolet radiation (UV-B) upon cuticular waxes in some crop plants. Planta. 1985;164:557-64.

16. Lavola A, Julkunen-Tiitto R, Aphalo P, Rosa TDL, Lehto T. The effect of u.v.-B radiation on U.v.-absorbing secondary metabolites in birch seedlings grown under simulated Forest soil conditions. New Phytol. 2010;137:617-21.

17. Jenkins GI. The UV-B photoreceptor UVR8: from structure to physiology. Plant Cell. 2014:26:21-37.

18. Kalbina I, Strid $\AA$. Supplementary ultraviolet-B irradiation reveals differences in stress responses between Arabidopsis thaliana ecotypes. Plant Cell Environ. 2006;29:754-63.

19. Hectors K, Prinsen E, De Coen W, Jansen MA, Guisez Y. Arabidopsis thaliana plants acclimated to low dose rates of ultraviolet $B$ radiation show specific changes in morphology and gene expression in the absence of stress symptoms. New Phytol. 2007;175:255-70.

20. Kliebenstein DJ, Lim JE, Landry LG, Last RL. Arabidopsis UVR8 regulates ultraviolet-B signal transduction and tolerance and contains sequence similarity to human regulator of chromatin condensation 1. Plant Physiol. 2002;130:234-43.

21. Wu D, Hu Q, Yan Z, Chen W, Yan C, Huang X, Zhang J, Yang P, Deng H, Wang J. Structural basis of ultraviolet-B perception by UVR8. Nature. 2012; 484:214-9.

22. Heijde M, UIm R. UV-B photoreceptor-mediated signalling in plants. Trends Plant Sci. 2012;17:230-7.

23. Di Ferdinando $M$, Brunetti $C$, Fini $A$, Tattini $M$. Flavonoids as antioxidants in plants under abiotic stresses. In: Ahmad P, Prasad MNV, editors. Abiotic stress responses in plants. New York: Springer; 2012. p. 159-79.

24. Day TA. Relating UV-B radiation screening effectiveness of foliage to absorbing-compound concentration and anatomical characteristics in a diverse group of plants. Oecologia. 1993;95:542-50.

25. Cloix C, Jenkins GI. Interaction of the Arabidopsis UV-B-specific signaling component UVR8 with chromatin. Mol Plant. 2008;1:118-28.

26. Brown BA, Jenkins GI. UV-B signaling pathways with different fluencerate response profiles are distinguished in mature Arabidopsis leaf tissue by requirement for UVR8, HY5, and HYH. Plant Physiol. 2008;146: $576-88$. 
27. Schatz MC, Witkowski J, McCombie WR. Current challenges in de novo plant genome sequencing and assembly. Genome Biol. 2012;13:243.

28. Pruitt KD, Tatusova T, Maglott DR. NCBI reference sequences (RefSeq): a curated non-redundant sequence database of genomes, transcripts and proteins. Nucleic Acids Res. 2006;35(Database issue):D61-5.

29. Huang C-H, Sun R, Hu Y, Zeng L, Zhang N, Cai L, Zhang Q, Koch MA, AlShehbaz I, Edger PP. Resolution of Brassicaceae phylogeny using nuclear genes uncovers nested radiations and supports convergent morphological evolution. Mol Biol Evol. 2015;33:394-412.

30. McKenzie R, Connor B, Bodeker G. Increased summertime UV radiation in New Zealand in response to ozone loss. Science. 1999;285:1709-11.

31. Gegas VC, Wargent JJ, Pesquet E, Granqvist E, Paul ND, Doonan JH. Endopolyploidy as a potential alternative adaptive strategy for Arabidopsis leaf size variation in response to UV-B. J Exp Bot. 2014;65:2757-66.

32. Sánchez-Bermejo E, Castrillo G, Del Llano B, Navarro C, Zarco-Fernández S, Martinez-Herrera DJ, Leo-del Puerto Y, Muñoz R, Cámara C, Paz-Ares J. Natural variation in arsenate tolerance identifies an arsenate reductase in Arabidopsis thaliana. Nat Commun. 2014:5:4617.

33. Gill SS, Anjum NA, Gill R, Jha M, Tuteja N. DNA damage and repair in plants under ultraviolet and ionizing radiations. Sci World J. 2015;2015:250158.

34. Heenan PB, Goeke DF, Houliston GJ, Lysak MA. Phylogenetic analyses of ITS and $r b c L$ DNA sequences for sixteen genera of Australian and New Zealand Brassicaceae result in the expansion of the tribe Microlepidieae. Taxon. 2012;61:970-9.

35. Greenberg BM, Wilson MI, Huang X-D, Duxbury CL, Gerhardt KE, Gensemer RW. The effects of ultraviolet-B radiation on higher plants. In: Gorsuch JW, Hughes JS, editors. Wuncheng W Wang, Plants for environmental studies. Florida: CRC Press; 1997. p. 1-35.

36. Li N, Teranishi M, Yamaguchi H, Matsushita T, Watahiki MK, Tsuge T, Li S-S, Hidema J. UV-B-induced CPD photolyase gene expression is regulated by UVR8-dependent and-independent pathways in Arabidopsis. Plant Cell Physiol. 2015;56:2014-23.

37. Jiang L, Wang Y, Björn LO, Li S. Arabidopsis radical-induced cell death1 is involved in UV-B signaling. Photoch Photobio Sci. 2009;8:838-46.

38. Muñoz MJ, Moreno NN, Giono LE, Botto AEC, Dujardin G, Bastianello G, Lavore S, Torres-Méndez A, Menck CF, Blencowe BJ. Major roles for pyrimidine dimers, nucleotide excision repair, and ATR in the alternative splicing response to UV irradiation. Cell Rep. 2017;18:2868-79.

39. Wargent J, Nelson B, McGhie T, Barnes P. Acclimation to UV-B radiation and visible light in Lactuca sativa involves up-regulation of photosynthetic performance and orchestration of metabolome-wide responses. Plant Cell Environ. 2015:38:929-40.

40. Gawel N, Jarret R. A modified CTAB DNA extraction procedure for Musa and Ipomoea. Plant Mol Biol Rep. 1991;9:262-6.

41. Cox MP, Peterson DA, Biggs PJ. SolexaQA: at-a-glance quality assessment of Illumina second-generation sequencing data. BMC bioinformatics. 2010;11:485.

42. Krueger F. Trim galore: a wrapper tool around Cutadapt and FastQC to consistently apply quality and adapter trimming to FastQ files. 2015. Available from http://www.bioinformatics.babraham.ac.uk/projects/trim_galore/.

43. Xie Y, Wu G, Tang J, Luo R, Patterson J, Liu S, Huang W, He G, Gu S, Li S. SOAPdenovo-trans: de novo transcriptome assembly with short RNA-Seq reads. Bioinformatics. 2014:30:1660-6.

44. Kajitani R, Toshimoto K, Noguchi H, Toyoda A, Ogura Y, Okuno M, Yabana M, Harada M, Nagayasu E, Maruyama H. Efficient de novo assembly of highly heterozygous genomes from whole-genome shotgun short reads. Genome Res. 2014. https://doi.org/10.1101/gr.170720.113.

45. Gurevich A, Saveliev V, Vyahhi N, Tesler G. QUAST: quality assessment tool for genome assemblies. Bioinformatics. 2013;29:1072-5.

46. Langmead B, Salzberg SL. Fast gapped-read alignment with bowtie 2. Nat Methods. 2012;9(4):357.

47. Haas BJ, Delcher AL, Mount SM, Wortman JR, Smith RK Jr, Hannick LI, Mait $\mathrm{R}$, Ronning CM, Rusch DB, Town CD. Improving the Arabidopsis genome annotation using maximal transcript alignment assemblies. Nucleic Acids Res. 2003;31:5654-66.

48. Simão FA, Waterhouse RM, loannidis P, Kriventseva EV, Zdobnov EM BUSCO: assessing genome assembly and annotation completeness with single-copy orthologs. Bioinformatics. 2015;31:3210-2.

49. Li H, Handsaker B, Wysoker A, Fennell T, Ruan J, Homer N, Marth G, Abecasis $G$, Durbin R. The sequence alignment/map format and SAMtools. Bioinformatics. 2009;25:2078-9.
50. Zhang G, Liu X, Quan Z, Cheng S, Xu X, Pan S, Xie M, Zeng P, Yue Z, Wang $W$. Genome sequence of foxtail millet (Setaria italica) provides insights into grass evolution and biofuel potential. Nat Biotechnol. 2012;30:549-54.

51. Smit A, Hubley R. RepeatModeler Open-1.0.8. 2008. Available from http:// www.repeatmasker.org/.

52. Xu Z, Wang H. LTR_FINDER: an efficient tool for the prediction of full-length LTR retrotransposons. Nucleic Acids Res. 2007;35(suppl_2):W265-8.

53. Haas B. TransposonPSI: an application of PSI-blast to mine (retro-) transposon ORF homologies. 2007. Available from http://transposonpsi. sourceforge.net/.

54. Han Y, Wessler SR. MITE-hunter: a program for discovering miniature inverted-repeat transposable elements from genomic sequences. Nucleic Acids Res. 2010;38:e199.

55. Kapitonov W, Jurka J. A universal classification of eukaryotic transposable elements implemented in Repbase. Nat Rev Genet. 2008:9:411-2.

56. Smit A, Hubley R, Green P. RepeatMasker Open-4.0.5. 2015. Available from http://www.repeatmasker.org/.

57. Campbell MS, Holt C, Moore B, Yandell M. Genome annotation and curation using MAKER and MAKER-P. Curr Protoc Bioinformatics. 2014; 48:4.11.11-14.11.39.

58. Stanke M, Diekhans M, Baertsch R, Haussler D. Using native and syntenically mapped cDNA alignments to improve de novo gene finding. Bioinformatics. 2008;24:637-44.

59. Altschul SF, Gish W, Miller W, Myers EW, Lipman DJ. Basic local alignment search tool. J Mol Biol. 1990;215:403-10.

60. Jones $\mathrm{P}$, Binns $\mathrm{D}$, Chang H-Y, Fraser M, Li W, McAnulla C, McWilliam H, Maslen J, Mitchell A, Nuka G. InterProScan 5: genome-scale protein function classification. Bioinformatics. 2014;30:1236-40.

61. Jin J, Tian F, Yang D-C, Meng Y-Q, Kong L, Luo J, Gao G. PlantTFDB 4.0: toward a central hub for transcription factors and regulatory interactions in plants. Nucleic Acids Res. 2017:45:D1040-5.

62. Moriya Y, Itoh M, Okuda S, Yoshizawa AC, Kanehisa M. KAAS: an automatic genome annotation and pathway reconstruction server. Nucleic Acids Res. 2007;35(suppl_2):W182-5.

63. Thiel T, Michalek W, Varshney R, Graner A. Exploiting EST databases for the development and characterization of gene-derived SSR-markers in barley (Hordeum vulgare L.). Theor Appl Genet. 2003:106:411-22.

64. Nawrocki EP, Eddy SR. Infernal 1.1: 100-fold faster RNA homology searches Bioinformatics. 2013:29:2933-5.

65. Goodstein DM, Shu S, Howson R, Neupane R, Hayes RD, Fazo J, Mitros T, Dirks W, Hellsten U, Putnam N. Phytozome. A comparative platform for green plant genomics. Nucleic Acids Res. 2011;40(D1):D1178-86.

66. Delcher AL, Salzberg SL, Phillippy AM. Using MUMmer to identify similar regions in large sequence sets. Curr Protoc Bioinformatics. 2003; 00:10.3.1-18.

67. Emms DM, Kelly S. OrthoFinder: solving fundamental biases in whole genome comparisons dramatically improves orthogroup inference accuracy. Genome Biol. 2015:16:157.

68. Cheng CY, Krishnakumar V, Chan AP, Thibaud-Nissen F, Schobel S, Town CD Araport11 a complete reannotation of the Arabidopsis thaliana reference genome. Plant J. 2017;9:789-804

69. Letunic I, Bork P. Interactive tree of life (iTOL) V3. an online tool for the display and annotation of phylogenetic and other trees. Nucleic Acids Res. 2016;44(W1):W242-5.

70. Wang Y, Coleman-Derr D, Chen G, Gu YQ. OrthoVenn: a web server for genome wide comparison and annotation of orthologous clusters across multiple species. Nucleic Acids Res. 2015;43(W1):W78-84.

71. Ignat T, Schmilovitch Z, Feföldi J, Bernstein N, Steiner B, Egozi H, Hoffman A. Nonlinear methods for estimation of maturity stage, total chlorophyll, and carotenoid content in intact bell peppers. Biosyst Eng. 2013;114:414-25.

72. Castells E, Molinier J, Drevensek S, Genschik P, Barneche F, Bowler C. det1-1induced UV-C hyposensitivity through UVR3 and PHR1 photolyase gene over-expression. Plant J. 2010;63:392-404.

73. Castelain M, Le Hir R, Bellini C. The non-DNA-binding bHLH transcription factor PRE3/bHLH135/ATBS1/TMO7 is involved in the regulation of light signaling pathway in Arabidopsis. Physiol Plantarum. 2012;145:450-60.

\section{Publisher's Note}

Springer Nature remains neutral with regard to jurisdictional claims in published maps and institutional affiliations. 